\title{
SOBRE LA ESFERA DE SIGNIFICACIÓN. REFLEXIONES EN TORNO A LA CUARTA INVESTIGACIÓN DE E. HUSSERL
}

\author{
Ariel O. Dottori \\ Universidad de Buenos Aires - CONICET
}

http://dx.doi.org/10.5209/rev_NOMA.2012.v34.n2.40735

\begin{abstract}
Resumen.- El interés de Husserl, en la presente Investigación, es establecer y aclarar una serie de distinciones; en primer lugar, la distinción entre expresiones categoremáticas y sincategoremáticas, cerradas y abiertas y, en segundo lugar, entre objetos independientes y no-independientes de la esfera de la significación. Oponiéndose a la gramática moderna, la cual se construye a partir de la psicología y demás disciplinas empíricas, Husserl rescata la vieja idea de una gramática general y apriorística debido a que, a diferencia de la anterior, logra demostrar que existen leyes apriorísticas que determinan las formas posibles de significación.
\end{abstract}

Palabras Clave.- Significaciones, gramática general, ciencia.

Abstract.- Husserl's interest in this research is to establish and clarify a number of distinctions: first, the distinction between categorematic and syncategorematic expressions, closed and open and, secondly, between dependent and independent objects, independent of the sphere of the significance. Opposing modern grammar, which is constructed from psychology and others empirical disciplines, Husserl rescued the old idea of a general grammar and aprioristic because, unlike the previous one, that can show that there are laws that determine a priori possible forms of meaning.

Keywords.- Significations, general grammar, science.

I.

Las significaciones compuestas se encuentran constituidas por significaciones simples. Mientras que las primeras son oraciones elementales, las segundas son las palabras que las componen. Así por ejemplo la significación, "un hombre más bueno que el pan" esta constituida por "hombre", "bueno", "pan". Pero a menudo, se presentan otras dificultades. La significación supuesta, ¿debe considerarse como simple o como compuesta?

A continuación, Husserl aborda el problema de los nombres propios. Si quisiéramos considerar las significaciones correspondientes a los nombres propios como simples nos encontraríamos con una dificultad. Con el nombre Rodríguez nos representamos un cierto hombre, nos lo representamos tanto como perteneciente a un hombre en general, como una multitud de rasgos individuales que distinguen a esa persona del resto. Pero por otro lado, es dificultoso el intento de hacer corresponder dentro de la significación propia, significaciones parciales a las determinaciones atributivas del objeto significado y representado; también es difícil admitir que esa significación propia sea idéntica a la significación compleja.

Una reflexión más detallada le permite a Husserl distinguir un doble sentido de la simplicidad y la composición. De éste modo, "la simplicidad, tomada en un sentido, no excluye la composición, tomada en otro sentido" (p. 440). La 
significación propia (en nuestro ejemplo, Rodríguez), si bien proporciona nuevas significaciones, es simple. Un nombre propio, usado con sentido, representa el objeto nombrado (la persona Rodríguez) como un determinado señor con un cierto contenido. Puesto que el contenido representativo no puede faltar por completo, la indeterminación jamás puede estar vacía de contenido. Ello es así porque, la conciencia, incluso la conciencia intuitiva, lleva consigo cierto contenido intencional.

En la conciencia de la significación de los nombres propios se revela una cierta bilateralidad, una cierta doble dirección; podemos hablar de composición o simplicidad. Uno de estos lados determina la composición o simplicidad de la significación misma. "Es, pues, el lado en el que reside la esencia intencional de la conciencia concreta y plena de la significación que, concebida específicamente, es la significación" (p. 441). En nuestro ejemplo del nombre propio, el lado es simple. Pero supone otro contenido intencional, puesto que lo mismo puede ser representado de un modo distinto. Las diferentes representaciones no afectan a la significación misma.

En el ejemplo dado del nombre propio (o significación propia) se ha dado un elemento por supuesto. Se trataba del nombre propio de una persona conocida. Esa es la razón por la cual funciona normalmente; no se la conoce en un sentido indirecto, como cierta persona llamada Rodríguez. Esta última significación sería, pues, compuesta (p.441).

Las significaciones compuestas nos son dadas regularmente como significaciones de complexiones verbales articuladas. Ante esta situación se plantean dos cuestiones. Por un lado, ¿a cada palabra de la complexión se le adscribe una significación?; por otro lado, ¿toda articulación y forma de expresión verbal vale como sello de una articulación o forma correspondiente de la significación? Si bien Bolzano le había adscripto a cada conjunción o proposición una significación, encontramos en un idioma palabras y expresiones que sólo son co-significativas, es decir, no poseen en sí mismas significación alguna y la adquieren en la conexión con otra significación. Hay expresiones completas e incompletas de representaciones, juicios, de fenómenos del sentimiento y de la voluntad; sobre esta distinción se fundan los conceptos del signo categoremático y sincategoremático.

\section{II.}

Contrariamente a lo sostenido por Bolzano, Husserl advierte que "...la articulación en la expresión no tiene la menor relación con articulaciones algunas en la significación. Las palabras sincategoremáticas, que ayudan a construir la expresión no tienen, pues, propiamente significación, y sólo la expresión completa tiene, en verdad, una significación"1 (p. 443).

\footnotetext{
${ }^{1}$ La posición de Husserl se asemeja, prima facie, a la propuesta por Frege, para quien la unidad mínima de significación no son las palabras sino la oración elemental. A partir de esta primer ruptura fregeana, le fue posible al Wittgenstein tardío elaborar una más radical; nos referimos a su noción de juego de lenguaje.
} 
En el parágrafo 5 Husserl introduce una nueva distinción. No sólo distingue entre expresiones categoremáticas y sincategoremáticas, sino también entre significaciones categoremáticas y sincategoremáticas. Pero serán empleados los términos independientes y no-independientes. Los sincategoremáticos son comprendidos incluso cuando se presentan solos; son depositarios de momentos de significación que reclaman cierto complemento, el cual se encuentra codeterminado por el contenido dado. Cuando, por el contrario, el sincategoremático funciona normalmente, esto es, cuando aparece en la conexión de una expresión independiente y conclusa, siempre tiene una relación determinada de significación con el pensamiento total.

Las expresiones sincategoremáticas son no-independientes y, por ello mismo, necesitan complementación; a ello se debe que también se las llame expresiones incompletas. Para clarificar la conceptualización, Husserl aclara que las significaciones independientes y no-independientes se cruzan con la división entre simples y compuestas. Significaciones tales como, por el nombre de Dios, ágil como un gato, a quien corresponda, bienvenido sea, son significaciones no independientes y unitarias -pese a la pluralidad de elementos diferenciables. Así, varias significaciones no-independientes 0 independientes en parte y en parte no-independientes, pueden entretejerse y formar unidades relativamente cerradas; al considerarlas como un todo, empero, tienen el carácter de significaciones no-independientes (p. 447). Cada unidad relativamente cerrada de expresiones compuestas sincategoremáticas es una expresión una, le pertenece una significación una, y es expresión compuesta porque da significación articulada a una expresión compuesta.

Con las expresiones anómalamente abreviadas sucede algo muy distinto. Estas dan al pensamiento una expresión incompleta pero, así y todo, se vuelven compresibles dadas las circunstancias del discurso. Las expresiones defectuosas son aquellas en las que faltan algunos miembros sintácticos. La necesidad de complemento en este último tipo de expresiones tiene un carácter muy distinto a la necesidad de complemento en los sincategoremáticos. Ello es así porque falta una significación unitaria; la oración defectuosa no puede funcionar como oración cerrada, ni aún siquiera como oración. Si desciframos una oración incompleta, tal como, Cesar... qui... duabus... (p. 447), podríamos contar con indicios externos que nos hagan suponer que se trata de una oración. Todos estos conceptos (expresiones sincategoremáticas, expresiones anómalamente abreviadas y las expresiones defectuosas que no son expresiones en sentido propio sino fragmentos de expresiones) se entrecruzan. Una expresión abreviada puede ser categoremática; una expresión sincategoremática puede ser completa y así sucesivamente.

\section{III.}

Hasta aquí Husserl ha demostrado que la distinción entre expresiones categoremáticas y sincategoremáticas es fundamental en la esfera de las significaciones. Aún cuando se haya tomado como punto de partida a las primeras, resultó que las últimas son primordiales, pues fundan aquella otra distinción gramatical. El concepto de expresión sólo puede ser fijado 
recurriendo a una diferencia entre las significaciones. Así, si éstas últimas se dividen en simples y compuestas, las expresiones también se dividen en adecuadas o simples o compuestas; esta composición conduce a la significación, sintaxis y, por lo tanto, a expresiones. Por el contrario, la división de las expresiones como meros fenómenos sensibles siempre da por resultado partes que también son meramente sensibles y no significativas.

Deteniéndose en la noción de significación sincategoremática, Husserl sostiene que, "...la designación de las significaciones sincategoremáticas como significaciones no-independientes indica ya en dónde colocamos nosotros la esencia de esas significaciones" (p. 448). Ya en sus ensayos sobre los contenidos no-independientes en general, Husserl había sostenido en general el concepto de la no-independencia; esa misma no-independencia es la que admitirá en la esfera de las significaciones ${ }^{2}$. Los contenidos no-independientes son aquellos que no pueden tener consistencia por sí mismos, sino sólo como partes de todos más amplios. Toda no-independencia obedece a una ley apriori según la cual, un contenido de la especie, por ejemplo, a, no puede existir más que en la conexión con un todo $T$. Al analizar específicamente la esfera de la significación y al tener en cuenta la división de los actos en simples y compuestos, puede un acto concreto contener varios actos parciales y éstos, a su vez, pueden residir en el todo, o bien como partes independientes, o bien como partes no-independientes. "Según esto, diremos que una significación es independiente cuando puede constituir la significación plena y total de un acto completo de significar. Y la llamaremos no-independiente cuando éste no sea el caso" (p. 449, cursivas del autor). La no-independencia así definida, determina la esencia de los sincategoremáticos.

A continuación, Husserl analiza la relación entre la independencia y noindependencia de las significaciones y la independencia y no-independencia de los objetos significados. Es falso suponer que las expresiones categoremáticas se refieren a objetos independientes y las sincategoremáticas a noindependientes. Todo objeto no-independiente admite ser objeto de una significación independiente, por ejemplo, la rojez, la figura, el ser, la igualdad, la unidad, el tamaño (p. 450). Es necesario aclarar que, si bien la significación "representa" un objeto, no se trata por ello de una copia; su esencia reside en el modo intencional de "dirigirse a" lo independiente como a lo noindependiente. De este modo, todo puede ser objeto intencional, todo puede ser objeto en el modo del significar.

Una explicación aparte merece la comprensión de los sincategoremáticos sueltos o desligados de todo enlace. Husserl sostiene que no puede haber tal comprensión; según ella, los elementos no-independientes de la oración cerrada categoremática son indesignables. Remitiéndose a la distinción entre representaciones "propias" e "impropias" (o lo que es lo mismo, entre significaciones meramente intencionales e impletivas), podría decirse que, "los sincategoremáticos sueltos, como por, en relación, con, $y$, o, etc., no pueden obtener una comprensión intuitiva, un cumplimiento significativo, como no sea en conexión con un todo de significación más amplio" (p. 451). Para esclarecer, por ejemplo, la significación de la palabra con, tendremos que efectuar un acto

\footnotetext{
${ }^{2}$ Ver al respecto la Investigación tercera, especialmente los parágrafos de 5 a 7 .
} 
de complementación y, en su conjunto, llevar a cabo una significación del tipo a con $b$; si queremos detenernos en la palabra $y$, tendremos que verificar una agregación del tipo a y $b$. Así sucesivamente.

De este modo, Husserl alcanza un resultado valioso: "ninguna significación sincate- goremática, esto es, ningún acto de intención significativa noindependiente, puede estar en la función de conocimiento, como no esté en la conexión de una significación categoremática" (p. 451, cursivas del autor). Pero, si ello es cierto, ¿cómo es posible que comprendamos las partículas $y$, con, aisladamente?, ¿las partículas aisladas son acaso un mero flatus vocis? La dificultad es resuelta por Husserl del siguiente modo.

El sincategoremático suelto, o bien no tiene la misma significación que unido, o bien la tiene. El con aislado lo comprendemos: o bien porque se asocia a él el pensamiento indirecto, no articulado en palabras, o bien porque se nos ofrece un pensamiento del tipo a con $b$, sin complemento verbal ni auxilio de vagas representaciones de objetos. En éste último caso la palabra con funciona normalmente, pues sólo pertenece a un momento de la intención significativa completa; pero, por otro lado, es anómala al no estar en conexión con otras expresiones.

Si la diferencia ente significaciones independientes y no-independientes se refiere a la diferencia más general entre objetos independientes y noindependientes, "las significaciones obedecen a leyes "a priori" que regulan su enlace en nuevas significaciones" (p. 452, cursivas del autor). A cada caso de significación no-independiente le corresponde una ley de esencia que regula su complemento con significaciones nuevas. Estas leyes de esencias (que son apriorísticas) actúan en todo enlace de significaciones en particular, y en todo enlace en general; así, todos los enlaces en general siguen leyes puras.

Refiriéndonos exclusivamente a la esfera de las significaciones, el enlace de una significación no obedece al capricho, no somos libres de llevarlo a cabo a nuestro antojo. Las significaciones sólo se unen, en ciertos modos determinados de antemano; las restantes posibilidades de enlace están excluidas por la ley, produciendo, de éste modo, una sarta de significaciones y no una significación unitaria. Esto implica que la imposibilidad de efectuar ciertos enlaces no obedece al ámbito de la subjetividad, sino más bien, es de carácter objetivo, ideal, fundada en la "naturaleza", en la esencia pura de la esfera de la significación, y debe ser aprehendida por evidencia apodíctica. Esta imposibilidad grava las categorías de significación (p. 455).

\section{IV.}

Como vemos, Husserl no ha dado cuenta únicamente de la imposibilidad, sino también de la posibilidad de los enlaces de significación. Tomemos como ejemplo la expresión: el pasto es verde; se trata de una expresión unitaria significativa. Al formalizarla lógicamente, adquiere la siguiente forma: $S$ es $p$. Si bien su particularización en proposiciones determinadas es posible de infinitos modos, no por ello somos totalmente libres; estamos ligados a limitaciones 
fijas. No toda significación puede ubicarse en el lugar de $S$ o $p$. Teniendo en cuenta la forma lógica, podemos sustituir el pasto es verde por: el agua, el perro, el número 2, la gaviota blanca, etc., es verde; es decir, podemos ubicar en lugar de $S$, cualquier materia nominal y, claro está, poner en lugar de $p$, cualquier materia adjetival. Donde corresponda una materia nominal, podrá estar otra materia nominal cualquiera, pero no una materia adjetival, o relacional, o proposicional; una materia de una categoría determinada, podrá ser reemplazada únicamente por otra materia de la misma categoría, pero no de otra. Esto vale para todas las significaciones.

Si bien es cierto que, al trocar materias dentro de su categoría, podrán aparecer significaciones ridículas, necias y falsas, necesariamente se tratarán de significaciones unitarias o expresiones gramaticales; si, en cambio, salimos de la categoría, ello no será posible. Las palabras, espumante en rojizo, exhaustivo es anguloso, este barco es igual, no son más que una sarta de palabras; cada palabra como tal tiene un sentido y remite a una conexión completa de sentido, pero no obtenemos en principio, un principio unitario cerrado. Nada prohíbe que hilvanemos palabras como, igual entre sueños, ayer o rojizo, excluyente ese árbol es y por lo tanto amargor, etc., pero esta sarta de palabras no es inteligible como una significación.

Es posible observar, a partir de los presentes análisis, que toda significación concreta es una reunión de materias y formas, que cada significación puede ser formalizada, y que a cada una de estas ideas corresponde una ley a priori de significación. Husserl sostiene que, "de aquí se deriva el gran problema, igualmente fundamental para la lógica y la gramática, de establecer esta constitución "a priori", que envuelve el reino de las significaciones, y de investigar en una "morfología de las significaciones" el sistema a priori de las estructuras formales, esto es, de las estructuras que prescinden de toda particularidad material de las significaciones" (p. 455, cursivas del autor).

En el parágrafo 11, Husserl se detiene a contrarrestar posibles objeciones. En primer lugar, no debemos dejarnos engañar cuando significaciones de categoría diversa, e incluso formas sincategoremáticas, como $y$, se coloquen en el lugar del sujeto, lugar que tiende a ser ocupado por significaciones sustantivas. Si se lo observa con detalle, esto sucede por medio de la modificación de la significación. A ello se debe que, por ejemplo, aquello que pasa al lugar del nombre sea en verdad un nombre, en vez de que sea utilizada otra figura sintáctica, tal como un adjetivo o una mera forma. Así ocurre en la expresión del tipo, "si" hay más de nueve planetas. Es evidente que la palabra "si" ocupa el lugar del sujeto, pero su significación no es la misma que aquella que le es propia en su significación normal. Aquello que le interesa a Husserl no es la composición de las palabras, sino de las significaciones. En términos lógicos, todo cambio de significación debe ser considerado anormal. De todos modos, los cambios de significación tienen lugar en todo idioma; por medio de la "conexión discursiva", la significación modificada puede ser comprendida fácilmente.

Toda expresión, categoremática o sincategoremática, se nombra a sí misma como fenómeno gramatical, se presenta como nombre de sí misma. "Si 
decimos: "la tierra es redonda" es un enunciado, funcionará como representación-sujeto, no la significación del enunciado, sino una representación del enunciado como tal. El juicio recaerá no sobre la situación objetiva de que la tierra es redonda, sino sobre la proposición enunciativa; y esta proposición misma funciona anómalamente como su propio nombre" (p. 456). Cuando decimos, " $y$ " es una conjunción (el ejemplo es del propio Husserl), no hemos colocado en el lugar del sujeto el momento de significación que usualmente le otorgamos a la palabra "y", sino que, muy por el contrario, habremos colocado en su lugar la significación independiente dirigida a la palabra "y". El "y", en ésta significación anómala, no es una palabra sincategoremática, sino una categoremática. Se nombra a sí misma.

Un caso análogo ocurre cuando una expresión no sustenta una significación normal, sino una representación de la significación. Ello sucede cuando decimos: "y", "pero", "mayor", son significaciones no independientes. En este caso diríamos, las significaciones de las palabras y, pero, mayor, son noindependientes. Así también, "hombre", "mesa", "caballo", son conceptos de cosas, funcionan como representaciones del sujeto, representaciones de esos mismos conceptos y no los conceptos mismos. En los ejemplos anteriores, se indica el cambio de significación utilizando las comillas (p. 456-57).

Cualquier significación puede experimentar a priori cierta transformación. "Existen en la esfera de las significaciones leyes apriorísticas, según las cuales algunas significaciones, conservando un núcleo esencial, se transforman de varios modos en nuevas significaciones" (p. 457, cursivas del autor). La significación modificada puede convertirse en la "representación directa" a ella referente, es decir, en la significación propia de la significación primitiva. Es así como, la expresión verbal funciona en la significación modificada como "nombre propio" de su significación primitiva. Esta modificación condiciona, tanto los equívocos generales gramaticales, como las modificaciones del significar verbal, que se encuentran en todos los idiomas empíricos particulares.

\section{V.}

Husserl se detiene a esclarecer una importante distinción sobre la que ya había avanzado en la Investigación primera. No se debe identificar lo que no tiene sentido (lo sin sentido) con lo que es absurdo (lo contra sentido). Si decimos cuadrado redondo, se nos ofrece una significación unitaria que en el "mundo" de las significaciones ideales tiene su "existencia", su modo de ser, pero, así y todo, no puede corresponder con ningún objeto existente. Si decimos, en cambio, vaya comió alas, o perro idéntico y, o cualquier sarta de palabras por el estilo, no existe significación alguna que se corresponda con el sentido expresado por estos enlaces. Toda expresión llevada a cabo en condiciones normales, tiende a evocar su significación; pero cuando una significación no se comprende, la sarta de palabras puede llegar a evocar una representación impropia de "cierta" significación correspondiente. Ello sucede porque echamos de menos la significación misma. 
En el caso de las significaciones sin sentido, tales como, los tres ángulos del cuadrado equilátero o, el hierro aguado, nos encontramos con nombres y proposiciones como cualquier otros pero las significaciones parciales no se corresponden en la unidad de significación; sus objetos referenciales sencillamente no existen. En el caso de lo contra sentido, muy por el contrario, "es la posibilidad misma de la significación unitaria la que no tolera que en ella coexistan ciertas significaciones parciales. Entonces poseemos sólo una representación indirecta, que apunta a la síntesis de dichas significaciones parciales en una significación única; y con ello poseemos al mismo tiempo la intelección de que a semejante representación no puede nunca corresponderle un objeto, esto es, de que una significación de la especie que aquí es intencionalmente referida, no puede existir" (p. 459-460).

Tanto las compatibilidades como las incompatibilidades apriorísticas analizadas hasta aquí por Husserl, hallan su expresión gramatical en las reglas que presiden el enlace gramatical de las partes integrantes de la oración. En un idioma, ciertos enlaces son prohibidos y otros permitidos. Y así ocurre gracias a los hábitos verbales accidentales y a la evolución lingüística que experimentan los distintos idiomas; pero además, aquello se debe a la diferencia esencial entre significaciones independientes y no-independientes, y a las leyes apriorísticas del enlace y transformación de las significaciones.

Una ciencia de las significaciones debería tener por objeto investigar la estructura legal esencial de las significaciones, las leyes del enlace de las significaciones en ella fundada y la modificación de las significaciones. Todas esas leyes, pues, deben reducirse al menor número de leyes independientes. Para estudiar, en un primer momento, las figuras de significación primitivas y sus estructuras internas, y determinar las puras categorías de significación, la aritmética es de suma utilidad, pues nos aclara esas leyes formales de enlace ${ }^{3}$. Existen ciertas formas de síntesis en las que, con cada dos números, se producen nuevos números. Tomemos como ejemplo las operaciones directas tales como, $a+b$ o $a b$, proporcionan una cantidad de números ilimitados como resultado. En el terreno de las significaciones también existen leyes semejantes, leyes que se refieren a la existencia de significaciones. En esas leyes, "las significaciones no son variables libres, sino que están limitadas a la extensión de unas u otras categorías fundadas en la naturaleza de la esfera de las significaciones" (pp. 460-61).

De aquí en más Husserl se detiene en el análisis de la lógica pura. Cito ad extensum, "En la lógica pura de las significaciones -cuyo fin supremo consiste en establecer leyes de validez objetiva de las significaciones, hasta donde esta validez venga condicionada por la pura forma de la significación-, el fundamento necesario lo constituye la teoría de la estructura esencial de las significaciones y leyes de sus formaciones" (p. 461). Aquello que la lógica tradicional no contempla son los fines que hay que poner bajo el punto de vista

\footnotetext{
${ }^{3}$ La preocupación por la aritmética también es compartida por Frege. El similar interés de ambos se advierte explícitamente en la carta de Frege a Husserl del 24 de Mayo de 1891 . No pretendo sugerir que el modo de abarcar la problemática de la relación entre lenguaje y aritmética (y mucho menos la terminología conceptual) sea la misma en ambos autores, pero sí sostengo que, tanto Frege como Husserl, se han acercado a problematizaciones similares.
} 
de la idea pura de la significación. En lugar de objetos materiales, entran representaciones universales indeterminadas de cosas en general.

Una morfología puramente lógica de las significaciones debe fijar, en primer lugar, las formas primitivas; en segundo lugar, las formas primitivas de la complicación y modificación; y, finalmente, la ilimitada variedad de las otras formas que se derivan de una mayor complicación o modificación. Aquellas formas que deben fijarse son las que, en particularizaciones arbitrarias proporcionan significaciones realmente existentes; a éstas formas Husserl las denomina "válidas". De este modo, a toda forma primitiva le pertenece cierta ley existencial apriorística que sostiene que "...todo enlace de significaciones, que sigue tal forma, da realmente una significación unitaria, con tal de que los términos (los indeterminados, los variables de la forma) pertenezcan a ciertas categorías de significación" (p. 461). A las formas derivadas, por su parte, les pertenecerán ciertas leyes existenciales que se deducen de las formas primitivas.

Así, por ejemplo, a cada dos significaciones nominales $M$ y $N$ pertenece la forma primitiva de enlace $M$ y $N$; el resultado del enlace es una significación de la misma categoría. La misma ley no sólo existe en el caso de las significaciones nominales, sino también en las significaciones proposicionales y adjetivales, por ejemplo. Dos proposiciones cualesquiera, que se unen bajo la forma $M$ y $N$, dan una proposición; dos adjetivos forman un adjetivo. Del mismo modo, a dos proposiciones cualesquiera, $M, N$, pertenecen las formas primitivas de enlace que siguen: si $M$, entonces $N o M$; de modo que el resultado es también una proposición. A toda significación nominal $S$, y a toda significación adjetival $p$, pertenece la forma primitiva $S p$ (por ejemplo, gato negro). El resultado que se ofrece es una nueva significación que pertenece a la categoría de la significación nominal. En las ideas categoriales de oración, representación nominal, representación adjetival, etc., lo que hacemos es una abstracción de las formas sintácticas cambiantes. Husserl aclara, "hablamos del mismo nombre, ya ocupe el puesto de sujeto, ya se halle en la función de objeto; hablamos del mismo adjetivo, ya funcione en el predicado o atributivamente; hablamos de la misma oración, ya se ofrezca como oración libre o como oración conjuntiva, disyuntiva, hipotética, ya sea miembro en éste o en aquel lugar de una oración compleja" (p. 462).

VI.

Ahora bien, si gradualmente enlazamos formas primitivas y aplicamos siempre la ley existencial primitiva, se irán creando nuevas formas de validez deductiva, enlazadas unas con otras, y aumentando el nivel de complejidad. De éste modo,

$(M$ y $N)$ y $P$

$(M$ y $N)$ y $(P$ y $Q)$

$[(M$ y $N)$ y $P]$ y $Q$ 
Y así sucesivamente. Siempre es posible agregar una complicación más, pues las combinaciones progresan in infinitum. A su vez, cada una de las uniones de significaciones, poseen un sentido unitario. De este modo, a la conciencia se le torna inteligible la constitución apriorística de la esfera de significación.

El único fin que persiguen las Investigaciones lógicas, radica en la "total intelección de la constitución formal de toda la esfera de la significación" ( $p$. 463). Husserl no pretende, empero, que la formulación de los tipos de significación y las leyes existenciales que a ellos pertenecen, puedan proporcionar reglas prácticas de la complexión de significaciones o de la complexión gramatical de expresiones. El interés de Husserl está motivado por el esclarecimiento de las leyes apriorísticas de la esfera de la significación.

Las leyes formales de la significación, que separan la esfera de lo que no tiene sentido de la que no lo tiene, valen como leyes formales lógicas. Tengamos en cuenta que aquí, Husserl utiliza la palabra sinsentido en los términos antes analizados, es decir, como una sarta de palabras, como por ejemplo, perro arbusto codeaba monje sin, las cuales no pueden ser comprendidas unitariamente. Si bien cada palabra tiene su sentido, en la unidad dejan de tenerlo. "Estas leyes del sentido -o, dicho normativamente, del vitando sinsentido- adscriben a la lógica las formas de significación en general posibles, cuyo valor objetivo debe ante todo determinar. Pero la lógica lo hace estableciendo las leyes (de otra especie) que separan el sentido formal congruente del sentido formal incongruente, del contrasentido formal" (p. 464, cursivas del autor).

También es necesario, sostiene Husserl, dar cuenta de la división entre el contrasentido material (sintético) y el contrasentido formal o analítico. En el primero pueden incurrir conceptos de cosas, como en el caso de las proposiciones geométricas puras que pueden ser falseadas; por ejemplo, el triángulo cuadrado. El contrasentido formal o analítico, por su parte, nos permite comprender toda incompatibilidad objetiva, fundada en la pura esencia de las categorías de significación, despreocupándose de la materia del conocimiento. Las leyes de la lógica, tales como el "tercero excluido", "principio de contradicción", "modus ponens", son leyes del vitando contrasentido formal. Estas, "...nos enseñan lo que merced a la "forma pura del pensar" vale para todo lo objetivo en general, esto es, lo que para la validez objetiva de las significaciones puede decirse a priori de toda materia de la objetividad significada, sobre la base de la pura forma de significación en que es pensada" (p. 465, los destacados le pertenecen al autor). Si estas leyes son violadas, resulta ya la falsedad.

Husserl, al limitarse al a priori que arraiga puramente en la esencia genérica de la significación en cuanto tal, reconoce la legitimidad de una gramática universal al modo en que fue concebida por el racionalismo de los siglos XVII y XVIII. Así como en el terreno de la lógica se distingue la "lógica pura" de la lógica empírica y práctica, así también, en la esfera gramatical se separa lo apriorístico (esto es, la "forma ideal" del lenguaje) de lo empírico. Lo empírico viene determinado por rasgos universales de la naturaleza humana y por las particularidades accidentales de la raza, la historia del pueblo, la experiencia 
individual de la vida del individuo; en lo apriorístico, en cambio, radica el interés científico y filosófico más alto.

No sólo puede, sino que también debe haber una gramática universal en un sentido más amplio, es decir, tomando en consideración la esfera de lo universal humano en sentido empírico. Pero el interés filosófico de Husserl lo conduce a separar cuidadosamente lo apriorístico de lo empírico, pues no deja de adherir a la sentencia de Kant, que sostiene que la ciencia no debe confundir sus límites. De éste modo, una gramática universal en ese sentido preciso, es una ciencia concreta que se nutre de los varios conocimientos que tienen su lugar teorético en ciencias teoréticas esencialmente diferentes, ya sean ciencias empíricas o apriorísticas (p. 466).

\section{A modo de conclusión.}

La idea fundamental que Husserl defiende en sus análisis lingüísticos es que un idioma, además de poseer fundamentos fisiológicos, psicológicos e histórico-culturales, tiene también fundamentos apriorísticos. "Estos últimos se refieren a las esenciales formas de significación y a las leyes apriorísticas de su complexión o modificación; y no es imaginable idioma alguno que no este codeterminado esencialmente por ese a priori" (p. 467, cursivas del autor).

La pura morfología de las significaciones, dentro de la lógica pura, se delimita como una esfera que, considerada en sí, es una, primera y fundamental. Es el esqueleto que todo idioma efectivamente rellena y reviste de material empírico; ello obedece, tanto a motivos generales humanos, como a motivos empírico accidentales y cambiantes. Siempre, todo idioma estará ligado a este esqueleto ideal. Concluyendo sus análisis de la presente investigación, Husserl hace hincapié en un punto fundamental: "todos los tipos de significación determinados en la morfología pura, investigados sistemáticamente en sus articulaciones y estructuras -las formas fundamentales de las oraciones, la oración categórica con sus múltiples figuras particulares y formas de articulación, los tipos primitivos de oraciones proposicionales complejas, como las unidades oracionales conjuntivas, disyuntivas, hipotéticas, o las diferencias de la universalidad y particularidad por un lado y de la singularidad por el otro, las sintaxis de la pluralidad, de la negación, de las modalidades, etc.-, son enteramente apriorísticas y están fundados en la esencia ideal de las significaciones como tales, como igualmente las figuras de significación que se producen según las leyes operativas de la complexión y modificación y según esas formas primitivas" (pp. 467, 68).

El gramático no puede contentarse con sostener nociones precientíficas acerca de las formas de significación o con las representaciones empíricas que la gramática histórica le proporciona; muy por el contrario, debe avanzar en el terreno de esta esfera fundamental de la lógica que Husserl denomina, gramática lógica-pura. 


\section{Bibliografía consultada.}

- (2007) Heidegger, Martín; El Ser y el Tiempo, ed. FCE, México.

- (2007a) Heidegger, Martín; Filosofía, ciencia y técnica, ed. Universitaria, Chile.

- (1990) Husserl, Edmund; El artículo de la Encyclopeadia Britannica. Seguido

de la versión de Ch. V. Salmon publicado por la Enciclopedia y del ensayo "El artículo de la Encyclopeadia Britannica de Husserl y las anotaciones de Heidegger al mismo" de Walter Bimel, ed. UNAM, México.

- (1962) Husserl, Edmund; Ideas relativas a una fenomenología pura y a una filosofía fenomenológica, ed. FCE, México.

- (2005) Husserl, Edmund; Ideas relativas a una fenomenología pura y a una filosofía fenomenológica. Libro segundo: Investigaciones fenomenológicas sobre la constitución, ed. FCE, México.

- (1976) Husserl, Edmund; Investigaciones lógicas, ed. Revista de Occidente, España.

- (2009) Husserl, Edmund; La crisis de las ciencias europeas y la fenomenología trascendental, ed. Prometeo, Bs. As.

- (1962) Husserl, Edmund; La filosofía como ciencia estricta, ed. Nova, Bs. As.

- (2004) Husserl, Edmund; Meditaciones Cartesianas, ed. FCE, México. 